\title{
Vaspin and its relation to cancer (Review)
}

\author{
SHUBHAM AGARWAL and SHREYA DESAI \\ Division of Internal Medicine, Chicago Medical School at \\ Rosalind Franklin University of Medicine and Science, North Chicago, IL 60064, USA
}

Received June 16, 2021; Accepted September 21, 2021

DOI: $10.3892 /$ wasj.2021.129

\begin{abstract}
Adipose tissue releases signaling molecules and hormones which are known as adipokines. Adipose tissue also provides a medium for low-grade inflammation which occurs from adipocyte hypertrophy with ensuing hypoxia and cell death. Cancers are known to arise in states of low-grade inflammation. There have been attempts to understand the role of adipokines in cancer pathogenesis and to establish their use as diagnostic and prognostic tools, as well as treatment targets. The present review article aims to highlight the role of vaspin in various types of cancer. Vaspin is known to inhibit apoptosis via the phosphatidylinositol-3-kinase/protein kinase B pathway, which has also been implicated in the development of colorectal cancer. The inhibition of the apoptosis of cells with malignant potential promotes tumor formation. At the same time, vaspin exhibits anti-inflammatory and insulin-sensitizing properties, which possibly confer its tumor inhibitory effects observed in endometrial cancer. However, its role either as a tumor promoter or inhibitor in other types of cancer in which it has not yet been studied would be difficult to predict.
\end{abstract}

\section{Contents}

1. Introduction

2. Data collection methods

3. Colorectal cancer

Correspondence to: Dr Shubham Agarwal, Division of Internal Medicine, Chicago Medical School at Rosalind Franklin University of Medicine and Science, 3333 Green Bay Road, North Chicago, IL 60064, USA

E-mail: shubhamagarwaldr@gmail.com

Abbreviations: kDa, kilodalton; MeSH, medical subject headings; TNM,tumor, nodes and metastases; PI3K, phosphatidylinositol-3-kinase; Akt, protein kinase B; HOMA-IR: homeostatic model assessment of insulin resistance; HCC, hepatocellular carcinoma; ROS, reactive oxygen species; FIGO, International Federation of Obstetrics and Gynecology staging system; BMI, body mass index; QUICKI, quantitative insulin-sensitivity check index; MAPK, mitogen-activated protein kinase; ERK, extracellular signal-regulated kinase

Key words: vaspin, adipokine, cancer, hepatocellular carcinoma, endometrial cancer, colorectal cancer
4. Hepatocellular carcinoma

5. Endometrial cancer

6. Other types of cancer

7. Other conditions

8. Conclusion

\section{Introduction}

Adipose tissue has been identified as a complex organ with endocrine, metabolic and immunoregulatory functions. Adipose tissue releases hormones and signaling molecules, known as adipokines, which function in an autocrine and paracrine manner (1). In obesity, adipocyte hypertrophy and hyperplasia alter the physiological microenvironment within adipocytes. This hyperadiposity causes a chronic low-grade inflammation resembling chronically injured tissue with immune cell infiltration and remodeling (2). In 1863, Virchow hypothesized that the origin of cancer was located at sites of chronic inflammation, partly based on the hypothesis that certain irritants cause tissue injury and cell proliferation occurs in the ensuing inflammatory environment. It has been observed that patients with obesity have a higher risk of developing cancer and also have increased mortality rates (3). One proposed mechanism is that of white adipose tissue hypertrophy and the adipocyte outgrowing its blood supply (4). The hypoxia of these cells leads to adipocyte stress, death and an adverse adipokine secretion profile. Adipocyte hypertrophy also leads to insulin resistance, and the established link is so substantial that an adipocyte size threshold has been identified for an increased risk of insulin resistance (5).

Hida et al (6) first identified a 47 kilodalton (kDa) molecule comprised of 415 amino acids termed vaspin (visceral adipose tissue-derived serpin) in the visceral adipose tissue of Otsuka Long-Evans Tokushima fatty rats at the age when obesity and insulin plasma concentrations peaked. This is a model of human type 2 diabetes that shares common components of the human metabolic syndrome, including abdominal obesity, insulin resistance, hypertension and dyslipidemia (6). From their experiments, Hida et al (6) concluded that vaspin exerts a protective effect against insulin resistance. Its increased levels suggest possible anti-protease properties, thereby improving insulin sensitivity (6). Adipokines may play a significant role in the regulation of cell growth, proliferation, cell cycle, angiogenesis, tumor growth and metastases. Vaspin is also known 
to cause an imbalance in favor of apoptosis inhibition via the phosphatidylinositol-3-kinase (PI3K)/protein kinase B (Akt) pathway (7). Several adipokines have been linked to various types of cancer with notable attempts made as regards their use as diagnostic tools, prognostic markers and therapeutic targets. The present review article aimed to shed light on available studies that have linked vaspin to various types of cancer.

\section{Data collection methods}

All articles reporting adipokines and various types of cancer were considered. The PubMed electronic database was searched using medical subject heading (MeSH) terms related to various adipokines, and benign and malignant tumors. Vaspin was first discovered in 2005 (6). Hence, search results were considered from 2005 onwards to select relevant articles. Manually searching reference lists supplemented this for additional studies. Similarly, the Google Scholar database was also manually searched. Studies listing adipokines other than vaspin were excluded, since different adipokines use distinct mechanisms in exerting their effects. Review articles on other adipokines were also excluded. An initial search revealed 4,380 articles; finally, 11 clinical and translational studies were included (Fig. 1 and Table I).

\section{Colorectal cancer}

According to the study search performed, a total of three studies were found that attempted to highlight the association between vaspin and colorectal cancer. Fazeli et al (8) examined vaspin levels in 39 patients who had biopsy-confirmed colorectal cancer compared with those in control subjects. They found that the vaspin, visfatin and omentin-1 levels were significantly higher in patients with colorectal cancer (8). This result was obtained without adjusting for variables; following the adjustment for variables such as age, body mass index (BMI) and waist-to-hip ratio. Additionally, when examining the levels of vaspin in different groups according to sex, no significant difference was found between males and females. They also found that adipocytokines were not related to the stage of colorectal cancer in these patients (8).

Another study comprised 41 patients with confirmed colorectal cancer and 27 healthy controls (9). The included patients either had colon cancer or rectal/sigmoid cancer at various stages. Of note, no difference was observed between the serum levels of vaspin among the healthy volunteers and study subjects. However, there was a difference in the median age of the study subjects to controls ( 55 vs. 43 years) and the study subjects were enrolled 2-4 weeks following the surgical or colonoscopic removal of the tumor. It is possible that the therapeutic intervention and time interval may have altered the serum vaspin levels in the study subjects compared with the time point when the tumor burden was higher (9).

Kan et al (10) examined the effects of tumor burden on vaspin expression and cancer prognosis in 89 patients with confirmed colorectal cancer. In patients with disease progression, the vaspin levels at the time of initial diagnosis were 40.1 vs. $27.4 \mathrm{pg} / \mathrm{ml}(\mathrm{P}=0.021)$ in patients who remained disease-free at the end of the study period. They also illustrated vaspin immunostaining imaging results in tumor burden subgroups according to disease progression and disease-free status. The results of their study revealed that patients with progressive disease had a higher vaspin expression at the start of the study irrespective of the tumor burden characteristics. That study highlighted that vaspin levels may be used as a potential marker for cancer progression and early cancer detection (10).

Although the underlying mechanisms remain to be fully elucidated, vaspin may possess anti-apoptotic properties (7). The lack of apoptosis of malignant cells may prevent them from dying. There are a number of mechanisms through which malignant cells can acquire a reduced apoptotic state. This can occur when there is an imbalance between pro- and anti-apoptotic proteins, as well as in instances where the reduced function of the caspase enzyme family, which is involved in the actual apoptosis of cells, provides the cells with their morphology, and impairs cell death signaling (11). Vaspin is known to cause an imbalance in favor of apoptosis inhibition via the PI3K/Akt pathway (Fig. 2) (7). This pathway has been implicated in the development of colorectal cancer (12).

\section{Hepatocellular carcinoma}

Adipokines have been linked to inflammation with eventual fibrotic, steatotic and angiogenic changes (13). These changes are mechanisms that underlie the development of chronic liver disease. Diseases, such as chronic hepatitis $\mathrm{C}$ induce metabolic alterations, including insulin resistance and contribute to hepatic steatosis. Kukla et al (14) demonstrated that vaspin was an independent predictor of the severity of liver fibrosis and positively correlated with the stage of fibrosis. In the case of no fibrosis or mild fibrosis limited to portal spaces, the serum vaspin level has been shown to be significantly decreased in patients with hepatitis $\mathrm{C}$ as compared with healthy controls. Insulin resistance, as noted with the homeostatic model assessment of insulin resistance (HOMA-IR) measurements, has also been shown to be significantly increased in patients with hepatitis C (14).

According to the search performed, two studies evaluated the relation of serum vaspin levels with hepatocellular carcinoma (HCC). The first study comprised 69 patients with cirrhosis and HCC. That study included patients with cirrhosis due to viral causes ( $81 \%$ had hepatitis C) and non-alcoholic fatty liver disease/non-alcoholic steatohepatitis with the majority of patients in Child-Pugh class A or B (15).

The serum vaspin levels were found to be higher in patients with HCC compared with healthy volunteers ( 0.18 vs. $0.10 \mathrm{ng} / \mathrm{ml} ; \mathrm{P}=0.05)$. Serum vaspin levels were also higher in patients with HCC due to viral causes compared with $\mathrm{HCC}$ due to non-viral causes ( 0.23 vs. $0.10 \mathrm{ng} / \mathrm{ml} ; \mathrm{P}=0.02)$. Vaspin levels were also higher in patients with chronic hepatitis $\mathrm{C}$ compared to those with liver disease due to non-viral causes $(0.41$ vs. $0.10 \mathrm{ng} / \mathrm{ml}, \mathrm{p}=0.003)$. No difference was noted in the serum vaspin levels between different groups according to Child-Pugh scores or Barcelona clinic liver cancer scores. The authors of that study demonstrated that vaspin levels did not differ in patients with higher or lower HOMA-IR scores, indicating that the increase in vaspin levels was primarily from the cancer and not a compensatory mechanism for insulin resistance (15). The increase in vaspin levels may also be a 
Table I. Summary of the studies comparing patients to healthy subjects and the inference of serum vaspin levels.

\begin{tabular}{lcll}
\hline Author/(Refs.), year of publication & No. of subjects & \multicolumn{1}{c}{ Type of condition } & Vaspin \\
\hline Jung et al $(7), 2011$ & N/A & Aortic endothelial cells & N/A \\
Fazeli et al $(8), 2013$ & 69 & Colorectal cancer & Higher \\
Erdogan et al $(9), 2016$ & 68 & Colorectal cancer & No difference \\
Kan et al $(10), 2020$ & 89 & Colorectal cancer & Higher \\
Pazgan-Simon et al $(15), 2020$ & 89 & Hepatocellular carcinoma & Higher \\
Skonieczna et al $(16), 2019$ & N/A & Hepatocellular carcinoma & N/A \\
Cymbaluk-Ploska et al $(17), 2018$ & 168 & Endometrial cancer & Lower \\
Erdogan et al $(18), 2013$ & 130 & Endometrial cancer & Lower \\
Jabbari et al $(20), 2015$ & 90 & Medullary thyroid cancer & Higher \\
Ginis et al $(21), 2016$ & 72 & Prolactinoma & Lower \\
Zhu et al $(22), 2013$ & N/A & Human osteoblasts & N/A
\end{tabular}

N/A, not available.

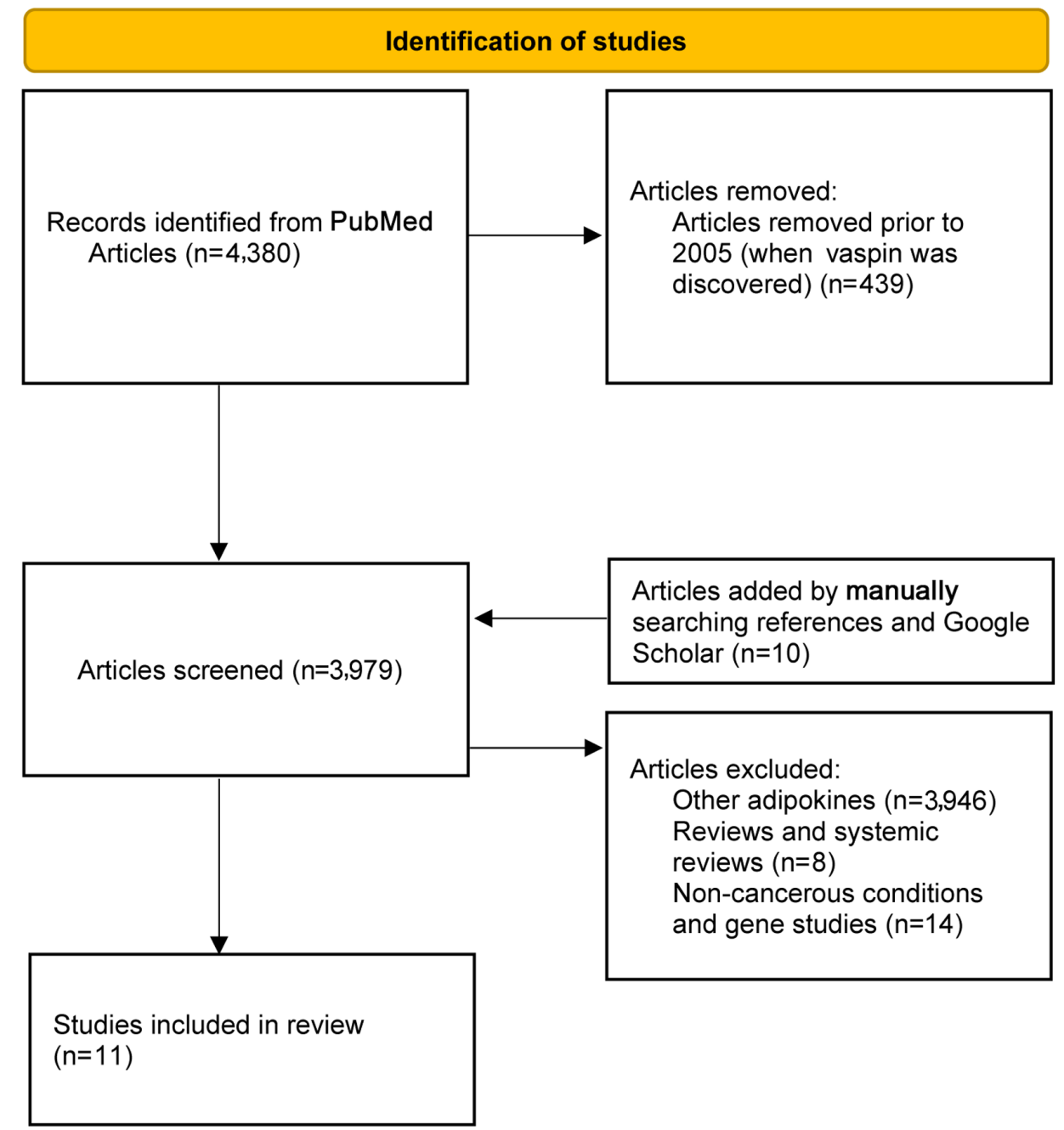

Figure 1. Flowchart of the study selection and criteria used for inclusion.

result of liver fibrosis, as demonstrated by Kukla et al (14), or a direct result of hepatitis $\mathrm{C}$ virus infection, which causes insulin resistance by affecting the insulin signaling pathway and inhibiting signal transduction.
The second study was performed by Skonieczna et al (16), in which human HCC cells treated with vaspin were cytometrically evaluated. The researchers demonstrated that vaspin reduced apoptosis in a concentration-dependent manner 


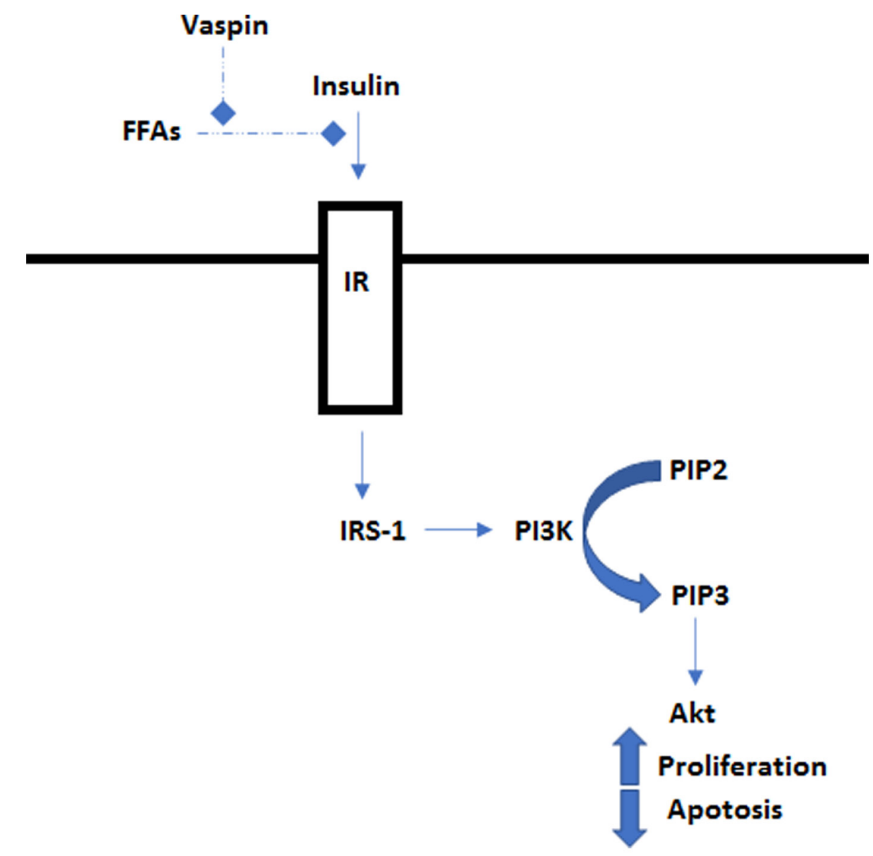

Figure 2. Insulin binds to the IR and activates IRS-1, which in turn activates PI3K. PI3K phosphorylates PIP2 to PIP3 which further activates Akt. Akt then regulates proteins involved in cell growth and proliferation. Vaspin inhibits the FFA-induced apoptosis via the PI3K/Akt pathway, increasing cell proliferation. FFA, free fatty acid, IR, insulin receptor; IRS-1, insulin receptor substrate 1; PI3K, phosphatidylinositol 3-kinase; PIP2, phosphatidylinositol-4,5-bisphosphate; PIP3, phosphatidylinositol-3,4,5-bisphosphate; Akt, protein kinase B

in the cancer cells, thus promoting the proliferation of tumor cells. They also noted that the production of reactive oxygen species (ROS), specifically nitric oxide and superoxide anion, which are commonly elevated in apoptotic cells and implicated in the promotion of apoptosis, was decreased in the presence of vaspin. The levels of ROS were related to vaspin in a concentration-dependent manner with a significant reduction when vaspin was used at $5 \mathrm{ng} / \mathrm{ml}$. The decreased levels of ROS observed with $5 \mathrm{ng} / \mathrm{ml}$ vaspin resulted from the suppression of physiological oxidative processes. The authors of that study suggested that an anti-apoptotic switch was activated at lower concentrations of vaspin and once initiated, the anti-apoptotic cascade was maintained with higher vaspin levels. The reduction in ROS generation could restrict pro-inflammatory processes in cancer cells, leading to increased viability and proliferation. Free radical scavenging in cells exposed to vaspin resulted in a reduction of pro-oxidative apoptotic signaling pathways (16).

\section{Endometrial cancer}

Chronic hyperinsulinemia is one of the main risk factors of endometrial cancer, and insulin is known to exhibit mitogenic and proliferative action through endometrial insulin receptors. Vaspin is known to play a role in augmenting tissue sensitivity to insulin, thereby protecting against one of the risk factors for endometrial cancer (6). It can be thus concluded that lower concentrations of vaspin would thus increase the risk of endometrial cancer development.

Following the search performed of the databases, two studies were found which investigated the relation of serum vaspin levels with endometrial cancer. In a previous study on 168 patients, including 92 patients with endometrial cancer and 76 patients with normal endometrium or benign polyps, no statistically significant differences in the mean values of vaspin levels were found in patients with endometrial cancer and patients with endometrial polyps or benign endometrium (17). However, significantly lower serum levels of vaspin were found in patients with lymph node or lymph vessel involvement. Lower levels were also observed in patients in whom the tumor had invaded deeper into the myometrium. The mean vaspin levels were examined between different tumor classification grades and were noted to be $1.1 \mathrm{ng} / \mathrm{ml}$ in patients with International Federation of Obstetrics and Gynecology (FIGO) stage I and II endometrial cancer, and $0.52 \mathrm{ng} / \mathrm{ml}$ in patients with FIGO stage III and IV endometrial cancer (17).

Another study by Erdogan et al examined at vaspin levels in 60 post-menopausal female patients with histologically confirmed endometrial cancer (18). The control group included 70 patients with benign endometrial conditions. The median vaspin levels were lower in patients with endometrial cancer compared with the controls ( 0.21 vs. $0.39 \mathrm{ng} / \mathrm{ml} ; \mathrm{P}<0.0001)$. A sub-group analysis of the various tertiles of vaspin levels also revealed that, when compared with the highest tertile of vaspin levels, the first tertile had an odds ratio of 17.04. This observation remained significant when the results were adjusted for BMI, HOMA-IR and the quantitative insulin-sensitivity check index (QUICKI) scores of the patients (18).

A possible mechanism of cancer development consists of understanding obesity and its implications. Obesity causes excess adipose tissue, which promotes the conversion of androgens to excess estrogen. Unopposed estrogen activity is known to be a risk factor of endometrial cancer. From cytological studies on vascular endothelial cells and smooth muscle cells, it is known that vaspin exerts an anti-inflammatory effect, thus protecting cells from tumorigenesis (19). This may explain why low vaspin levels were associated with a higher risk of developing endometrial cancer in both the studies on endometrial cancer mentioned above.

\section{Other types of cancer}

Jabbari et al (20) attempted to examined vaspin levels in medullary thyroid cancer in 45 patients with cancer and 45 healthy controls who were matched for age, sex and BMI. They found that the vaspin levels were elevated in patients with cancer compared with the healthy controls ( $0.52 \mathrm{vs} .0 .45 \mathrm{ng} / \mathrm{ml}$; $\mathrm{P}=0.0241$ ).

Another study examined 42 patients who were diagnosed with prolactinomas and compared them with 30 controls (21). They further subdivided the patients with prolactinoma into 21 patients who underwent treatment with dopamine agonists and 21 patients who were newly diagnosed and were treatment-naive. Patients with prolactinomas had higher HOMA-IR and QUICKI scores compared with the healthy volunteers. The mean vaspin levels were lower in the untreated and treated patients with prolactinoma compared with the healthy controls. In a subset analysis of the healthy controls, after categorizing the controls into two groups with the median value of serum vaspin, lower vaspin levels were found to be associated with an increased risk of prolactinoma (odds ratio, 6.00). These results 
did not change after adjusting for confounding factors, such as BMI, HOMA-IR and QUICKI scores (21).

\section{Other conditions}

Zhu et al (22) examined the effect of vaspin on human osteoblasts in relation to apoptosis. They noted that there was a consistent decrease in apoptosis with increasing concentrations of vaspin at up to $100 \mathrm{ng} / \mathrm{ml}$. Vaspin exposure also increased Bcl-2 protein expression and decreased Bax protein expression in a concentration-dependent manner. Bcl-2 protein is an inhibitor of apoptosis, while Bax protein is an inducer of apoptosis. With western blot analysis, the authors of that study were also able to demonstrate that vaspin exerted its anti-apoptotic effects by activating the mitogen-activated protein kinase (MAPK)/extracellular signal-regulated kinase (ERK) signaling pathway in human osteoblasts (22). Apoptosis is a natural phenomenon that occurs in aging cells. The anti-apoptotic activity of vaspin has been previously highlighted in HCC (16) and can be similarly responsible for the development of bone-related cancers.

Jung et al (7) established the anti-apoptotic nature of vaspin in endothelial cells. They studied the effects of vaspin on human aortic endothelial cells and discovered that free fatty acid-induced apoptosis was partly reversible with vaspin. In the vascular endothelium, there exists a signaling pathway that is very similar to that used by insulin to promote glucose transporter type 4 expression on the cell surface. The similar signaling pathway, when activated, increases nitric oxide production, which reduces endothelial dysfunction. Vaspin was noted to use the PI3K/Akt pathway to increase nitric oxide production. It also inactivated Bad protein (pro-apoptotic) and relieved the inhibitory effect on Bcl-2 protein (anti-apoptotic) with the increased expression of Akt and $\mathrm{Bcl}-2$, as recorded in that study (7).

\section{Conclusion}

Vaspin appears to play a role in the promotion of tumor formation, possibly by inhibiting apoptosis via cellular mechanisms. In turn, it can promote the proliferation of cells with malignant potential. Mechanisms involved in insulin resistance may be involved. Thus far, an attempt has been made to establish its role in colorectal cancer and HCC, albeit in a limited number of studies. However, vaspin also appears to exert anti-inflammatory effects via similar cellular signaling pathways, as highlighted in the studies on endometrial cancer mentioned above. It is difficult to predict whether vaspin functions as a tumor promoter or a tumor inhibitor in other types of cancer, as only a limited number of studies have explored its role in various therapeutics. Further studies are thus warranted to fully elucidate its potential as a possible diagnostic tool, prognostic marker, or therapeutic target.

\section{Acknowledgements}

Not applicable.

\section{Funding}

No funding was received.

\section{Availability of data and materials}

Not applicable.

\section{Authors' contributions}

SA was involved in the conceptualization, methodology and literature search for the study, as well as in the writing of the original draft. SD was involved in the literature search for the study, and in writing, editing and revision of the manuscript. SA and SD confirm the authenticity of all raw data. Both authors have read and approved the final manuscript.

\section{Ethics approval and consent to participate}

Not applicable.

\section{Patient consent for publication}

Not applicable.

\section{Competing interests}

The authors declare that they have no competing interests.

\section{References}

1. Booth A, Magnuson A, Fouts J and Foster M: Adipose tissue, obesity and adipokines: Role in cancer promotion. Horm Mol Biol Clin Investig 21: 57-74, 2015.

2. Iyengar NM, Gucalp A, Dannenberg AJ and Hudis CA: Obesity and cancer mechanisms: Tumor microenvironment and inflammation. J Clin Oncol 34: 4270-4276, 2016.

3. Calle EE, Rodriguez C, Walker-Thurmond $\mathrm{K}$ and Thun MJ: Overweight, obesity, and mortality from cancer in a prospectively studied cohort of U.S. adults. N Engl J Med 348: 1625-1638, 2003.

4. Fuster JJ, Ouchi N, Gokce N and Walsh K: Obesity-induced changes in adipose tissue microenvironment and their impact on cardiovascular disease. Circ Res 118: 1786-1807, 2016.

5. Cotillard A, Poitou C, Torcivia A, Bouillot JL, Dietrich A, Klöting N, Grégoire C, Lolmede K, Blüher M and Clément K: Adipocyte size threshold matters: Link with risk of type 2 diabetes and improved insulin resistance after gastric bypass. J Clin Endocrinol Metab 99: E1466-E1470, 2014.

6. Hida K, Wada J, Eguchi J, Zhang H, Baba M, Seida A, Hashimoto I, Okada T, Yasuhara A, Nakatsuka A, et al: Visceral adipose tissue-derived serine protease inhibitor: A unique insulin-sensitizing adipocytokine in obesity. Proc Natl Acad Sci USA 102: 10610-10615, 2005.

7. Jung CH, Lee WJ, Hwang JY, Seol SM, Kim YM, Lee YL and Park JY: Vaspin protects vascular endothelial cells against free fatty acid-induced apoptosis through a phosphatidylinositol 3-kinase/Akt pathway. Biochem Biophys Res Commun 413: 264-269, 2011.

8. Fazeli MS, Dashti H, Akbarzadeh S, Assadi M, Aminian A, Keramati MR and Nabipour I: Circulating levels of novel adipocytokines in patients with colorectal cancer. Cytokine 62: 81-85, 2013.

9. Erdogan S, Yilmaz FM, Yazici O, Yozgat A, Sezer S, Ozdemir N, Uysal S, Purnak T, Sendur MA and Ozaslan E: Inflammation and chemerin in colorectal cancer. Tumour Biol 37: 6337-6342, 2016.

10. Kan JY, Lee YC, Lin YD, Ho WY and Moi SH: Effect of baseline characteristics and tumor burden on vaspin expression and progressive disease in operable colorectal cancer. Diagnostics (Basel) 10: 801, 2020.

11. Wong RS: Apoptosis in cancer: from pathogenesis to treatment. J Exp Clin Cancer Res 30: 87, 2011.

12. Danielsen SA, Eide PW, Nesbakken A, Guren T, Leithe E and Lothe RA: Portrait of the PI3K/AKT pathway in colorectal cancer. Biochim Biophys Acta 1855: 104-121, 2015. 
13. Kukla M, Mazur W, Bułdak RJ and Zwirska-Korczala K: Potential role of leptin, adiponectin and three novel adipokines visfatin, chemerin and vaspin--in chronic hepatitis. Mol Med 17: $1397-1410,2011$.

14. Kukla M, Waluga M, Sawczyn T, Berdowska A, Kajor M, Boryczka G, Stygar D, Gabriel A, Zwirska-Korczala K and Hartleb M: Serum vaspin may be a good indicator of fibrosis in chronic hepatitis $\mathrm{C}$ and is not altered by antiviral therapy. Pol J Pathol 63: 213-220, 2012.

15. Pazgan-Simon M, Kukla M,Zuwała-Jagiełło J, Derra A, Bator M, Menżyk T, Lekstan A, Grzebyk E and Simon K: Serum visfatin and vaspin levels in hepatocellular carcinoma (HCC). PLoS One 15: e0227459, 2020

16. Skonieczna M, Hudy D, Hejmo T, Buldak RJ, Adamiec M and Kukla M: The adipokine vaspin reduces apoptosis in human hepatocellular carcinoma (Hep-3B) cells, associated with lower levels of NO and superoxide anion. BMC Pharmacol Toxicol 20: 58, 2019.

17. Cymbaluk-Płoska A, Chudecka-Głaz A, Jagodzinska A, Pius-Sadowska E, Sompolska-Rzechuła A, Machaliński B and Menkiszak J: Evaluation of biologically active substances promoting the development of or protecting against endometrial cancer. Onco Targets Ther 11: 1363-1372, 2018.

18. Erdogan S, Sezer S, Baser E, Gun-Eryilmaz O, Gungor T, Uysal S and Yilmaz FM: Evaluating vaspin and adiponectin in postmenopausal women with endometrial cancer. Endocr Relat Cancer 20: 669-675, 2013.
19. Phalitakul S, Okada M,Hara Y and Yamawaki H: Vaspin prevents TNF- $\alpha$-induced intracellular adhesion molecule-1 via inhibiting reactive oxygen species-dependent $\mathrm{NF}-\kappa \mathrm{B}$ and $\mathrm{PKC} \theta$ activation in cultured rat vascular smooth muscle cells. Pharmacol Res 64: 493-500, 2011.

20. Jabbari S, Hedayati M, Yaghmaei P and Parivar K: Medullary thyroid carcinoma - Circulating status of vaspin and retinol binding protein-4 in Iranian patients. Asian Pac J Cancer Prev 16: 6507-6512, 2015.

21. Ginis Z, Ucar F, Erdogan S, Ozturk G, Akyol S, Erden G, Arslan MS and Delibasi T: Serum vaspin and adiponectin levels in patients with prolactinoma. Scand J Clin Lab Invest 76: 17-24, 2016.

22. Zhu X, Jiang Y, Shan PF, Shen J, Liang QH, Cui RR, Liu Y, Liu GY, Wu SS, Lu Q, et al: Vaspin attenuates the apoptosis of human osteoblasts through ERK signaling pathway. Amino Acids 44: 961-968, 2013.

This work is licensed under a Creative Commons Attribution-NonCommercial-NoDerivatives 4.0 International (CC BY-NC-ND 4.0) License. 